\title{
MICROWAVE IMAGING REFLECTOMETRY FOR THE VISUALIZATION OF TURBULENCE IN TOKAMAKS
}

\author{
E. Mazzucato \\ Princeton Plasma Physics Laboratory, Princeton University, \\ P. O. Box 451, Princeton, New Jersey 08543
}

\begin{abstract}
Understanding the mechanism of anomalous transport in magnetically confined plasmas requires the use of sophisticated diagnostic tools for the measurement of short-scale turbulent fluctuations. This paper describes the conceptual design of an experimental technique for the global visualization of density fluctuations in tokamaks. The proposed method is based on microwave reflectometry and consists in using a large diameter probing beam, collecting the reflected waves with a large aperture antenna, and forming an image of the reflecting plasma layer onto a 2D array of microwave receivers. Based on results from a series of numerical simulations, the theoretical feasibility conditions of the proposed method are discussed.
\end{abstract}

Key words: Tokamak, anomalous transport, turbulent fluctuations, microwave imaging reflectometry. 


\section{Introduction}

The direct impact of plasma confinement on the feasibility of an economical fusion reactor makes understanding the mechanism of anomalous transport in magnetically confined plasmas one of the great challenges of fusion research.

Both theory and experiments suggest that plasma transport in tokamaks greatly exceeds neoclassical predictions because of short-scale turbulent fluctuations causing an enhancement in the diffusion of particles, energy and momentum across the magnetic field lines [1]. However, such an explanation is not completely satisfactory since it is based neither on a self-consistent theory of plasma turbulence nor on comprehensive sets of measurements. Indeed, the role played by turbulence on transport of magnetically confined plasmas is still an outstanding issue.

Because of the overwhelming difficulty in developing the theory of plasma turbulence and in performing exhaustive measurements of turbulent fluctuations, numerical simulations of turbulence are beginning to play a dominant role in the prediction and interpretation of tokamak experiments. However, this also is not satisfactory since, given the enormous complexity of the problem, any simulation of turbulence must be driven by a direct experimental observation of the turbulent fluctuations. A case in point is that of classical fluids [2], where many advances in the theory of hydrodynamic turbulence were stimulated by the visualization of the turbulent flow with a variety of optical techniques. Unfortunately, none of these diagnostic methods could be used for the measurement of fluctuations in low density and high temperature plasmas, such as those in tokamaks.

This paper describes an experimental technique for the global visualization of turbulent fluctuations in tokamaks. Its outline is as follows. Section 2 is a discussion of difficulties and limitations in the use of microwave reflectometry in tokamaks - the basis of the method 
proposed in this paper. Section 3 contains results from a series of numerical simulations, and their implications for the use of reflectometry in tokamaks are discussed in Sec. 4. Section 5 describes the conceptual design of a possible apparatus for the global visualization of turbulent fluctuations in tokamaks. Finally, concluding remarks are given in Sec. 6.

\section{Microwave Reflectometry}

The method described in this paper is based on microwave reflectometry [3] - a radar technique for the detection of plasma fluctuations from the reflection of microwaves by plasma cutoffs. Because of a high sensitivity to plasma fluctuations, reflectometry has found extensive use in tokamaks for the detection of turbulence. However, often the high sensitivity makes very difficult the extraction of any quantitative information from the measured signals as well.

The interpretation of reflectometry is relatively simple in a $1 \mathrm{D}$ geometry, where a plane stratified plasma permittivity $\varepsilon=\varepsilon_{0}(r)+\tilde{\varepsilon}(r)$ (with fluctuation component $\tilde{\varepsilon}(r)<<1$ ) is probed by a wave propagating in the $r$-direction. Under these conditions, it is easy to show that when the radial wave number of fluctuations satisfies the equation $k_{r}<k_{0} /\left(k_{0} L_{\varepsilon}\right)^{1 / 3}$ (where $L_{\varepsilon}=\left(d \varepsilon_{0} / d r\right)_{r=r_{C}}^{-1}$ is the scale length of the plasma permittivity at the cutoff $r=r_{c}$ and $k_{0}$ is the free-space wave number of the probing wave), the fluctuating component of the signal phase is given by the approximation of geometric optics [3]

$$
\tilde{\phi}=k_{0} \int_{0}^{r_{c}} \frac{\tilde{\varepsilon}(r)}{\sqrt{\varepsilon_{0}(r)}} .
$$

Taking $\left|k_{r}\right|>1 / L_{\varepsilon}$ since we are interested in short-scale fluctuations, and $\varepsilon_{0}(r) \approx\left(r_{c}-r\right) / L_{\varepsilon}$ since most of the contribution to $\tilde{\phi}$ comes from a narrow region near the cutoff, from Eq. (1) we obtain [3]

$$
\Gamma_{\phi}\left(k_{r}\right)=\pi M \frac{k_{0}^{2} L_{n}}{\left|k_{r}\right|} \Gamma_{n}\left(k_{r}\right),
$$


where $L_{n}=n /(d n / d r)_{r=r_{c}}$ is the scale length of the electron density $n$, $M \equiv(n \partial \varepsilon / \partial n)_{r=r_{C}}$ (equal to one for the ordinary mode and to two for the extraordinary mode), $\Gamma_{\phi}\left(k_{r}\right)$ is the power spectrum of $\tilde{\phi}$ (considered as a function of $r_{c}$ ) and $\Gamma_{n}\left(k_{r}\right)$ is the power spectrum of the relative density fluctuation $\tilde{n} / n$.

In conclusion, for 1D turbulence the power spectrum of density fluctuations $\Gamma_{n}\left(k_{r}\right)$ can be obtained from the power spectrum of the signal phase $\Gamma_{\phi}\left(k_{r}\right)$. The latter can be measured by performing radial correlation measurements using several probing waves with closely spaced cutoff layers.
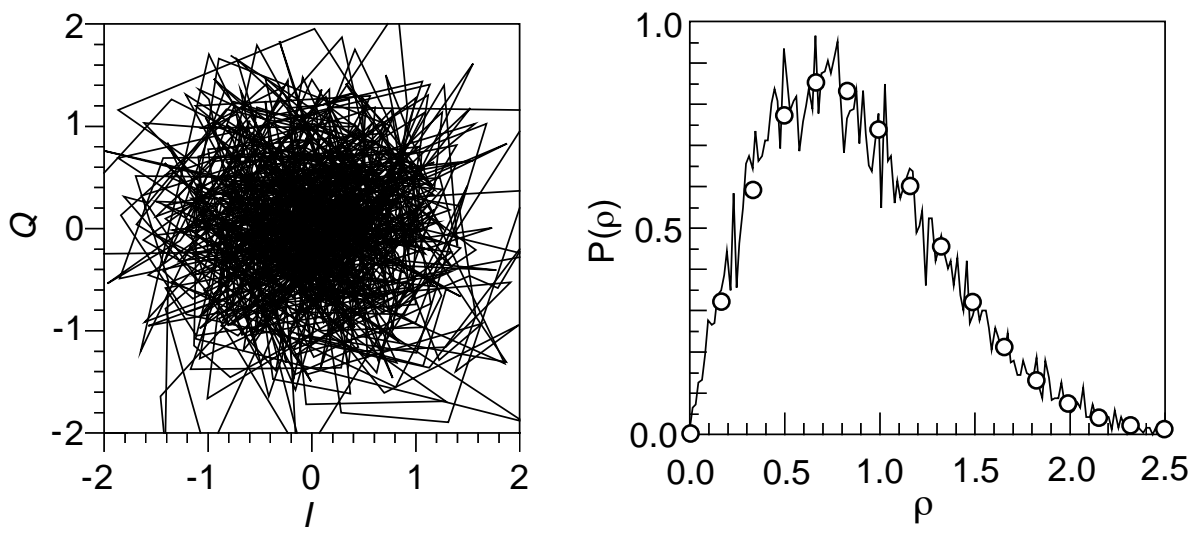

FIG. 1. In-phase $(I)$ and quadrature $(Q)$ components of a TFTR reflectometer signal over a $1 \mathrm{~ms}$ window (left), and probability density distribution of the signal amplitude $\rho=\sqrt{I^{2}+Q^{2}}$ (right); open circles are the Rayleigh distribution $\left(=2 \rho \exp \left[-\rho^{2}\right]\right)$.

The use of reflectometry becomes considerably more difficult in plasmas with multi-dimensional turbulent fluctuations. Unfortunately, this is just the case of interest in magnetically confined plasmas, such as in tokamaks where turbulent fluctuations vary in both the radial and poloidal directions. The difficulty stems from the fact that when the plasma permittivity fluctuates perpendicularly to the direction of propagation of the probing wave, the spectral components of the backward field propagate in different directions. This may result in a 
complicated interference pattern on the detector plane, from which it is very difficult to extract any information on the fluctuations under investigation. This phenomenon is illustrated in Fig. 1, which shows the in-phase $(I)$ and quadrature $(Q)$ components of a TFTR reflectometer signal together with the density distribution of the signal amplitude

$\rho=\sqrt{I^{2}+Q^{2}}$ [4]. The large fluctuations in the measured signal are the result of two dimensional (2D) plasma fluctuation that transforms the signal components into two independent normal random variables with zero mean. Consequently, the phase of the measured signal cannot be used for inferring the properties of plasma fluctuations.

To better understand and quantify this phenomenon, we have performed a series of numerical simulations of reflectometry in plasmas with 2D fluctuations [5]. Here we present only the results that are of interest for the subject of this paper.

\section{Numerical Simulations}

In a system of orthogonal coordinates $(x, r)$, we assume that a plane stratified plasma density $(n(r))$ is perturbed by a field of 2D fluctuations $(\tilde{n}(x, r))$ with spectral distribution

$$
\frac{\tilde{n}(x, r)}{n(r)}=\sum_{p=1}^{M} \sum_{q=1}^{M} \delta_{p q} \cos \left(p \kappa_{r} r\right) \cos \left(q \kappa_{x} x+\varphi_{p q}\right),
$$

consisting of $M X M$ discrete components with wave numbers $p \kappa_{r}$ and $q \kappa_{x}$ ( $\kappa_{r}$ and $\kappa_{x}$ are constants), random phases $\varphi_{p q}$, and amplitudes

$$
\delta_{p q}^{2} \propto p \exp \left[-\left(p \kappa_{r} / \Delta k_{r}\right)^{2}-\left(q \kappa_{x} / \Delta k_{x}\right)^{2}\right],
$$

where $\Delta k_{r}=\kappa_{r} M / 2, \Delta k_{x}=\kappa_{x} M / 2$. Throughout the paper we will use the value $M=20$.

The plasma is confined to the region $r<r_{b}$ and the probing wave is launched in the $r$-direction from the free-space region $r>r_{b}$. For facilitating the comparison with experimental results, the density $n(r)$ is taken similar to the electron density distribution on the equatorial plane 
of a typical TFTR discharge. Finally, the probing wave has the frequency of $75 \mathrm{GHz}$ and the ordinary mode of propagation with the electric field perpendicular to the $x$-axis.

The wave amplitude $(E(x, r))$ is expressed as the sum of $2 N+1$ independent solutions of the wave equation

$$
E(x, r)=\sum_{n=-N}^{N} c_{n} E_{n}(x, r),
$$

with $N>>M$ (to be determined). The functions $E_{n}$ are cast in the form

$$
E_{n}(x, r)=\sum_{m=-N}^{N} f_{m n}(r) e^{i m \kappa_{x} x}
$$

where $f_{m n}(r)$ are solutions of a system of $2 N+1$ ordinary differential equations

$$
\begin{aligned}
& \frac{d^{2} f_{m n}}{d r^{2}}+k_{0}^{2}\left(\varepsilon_{0}-\alpha_{m}^{2}\right) f_{m n}+k_{0}^{2}\left(\varepsilon_{0}-1\right) \\
& \mathrm{x} \sum_{p=1}^{M} \sum_{q=1}^{M}\left[\frac{\delta_{p q}}{2} \cos \left(p \kappa_{r} r\right)\left(f_{(m-q) n} e^{i \varphi_{p q}}+f_{(m+q) n} e^{\left.-i \varphi_{p q}\right)}\right]=0\right. \\
& \quad(m=-N,-N+1 \ldots \ldots . . N)
\end{aligned}
$$

where $\varepsilon_{0}=1-\left(\omega_{p} / \omega\right)^{2}$ is the unperturbed plasma permittivity, $\omega_{p}=\left(4 \pi n_{e} e^{2} / m_{e}\right)^{1 / 2}$ is the plasma frequency and $\alpha_{m}=m \kappa_{x} / k_{0}$. These equations, that are obtained inserting Eqs. (4) and (6) into the wave equation and by performing a Fourier expansion in $x$, can be solved with the Runge-Kutta method. The coefficients $c_{n}$ in Eq. (5) are then determined by imposing that $E(x, r)$ in free-space has the form

$$
E(x, r)=e^{-i k_{0} r}+\sum_{n=-N}^{N} A_{n} e^{i\left[n \kappa_{x} x+\left(k_{0}^{2}-n^{2} \kappa_{x}^{2}\right)^{1 / 2} r\right]}
$$

where the first term on the right hand side is the launched wave, while the second represents the field of reflected waves in free-space (in the following referred to as the backward field $E_{b}$ ). In the region $r<r_{b}, E_{b}$ represents a virtual field that an observer in free-space could measure by mapping the plasma region onto an array of detectors with an optical 
system.

Finally, the integer $N$ must be chosen large enough to make the results significantly unchanged by any increase in its value. This condition, to be verified a posteriori, allows the closure of the system of differential equations (7) by setting to zero all terms $f_{(m \pm q) n}$ with $|m \pm q|>N$.

Shown in Fig. 2 are the amplitudes $\left|A_{n}\right|$ of reflected waves as a function of $k_{x}=n \kappa_{x}$ for fluctuations with $\Delta k_{x}=0.5 \mathrm{~cm}^{-1}$ and $\Delta k_{r}=1$ $\mathrm{cm}^{-1}$. The three cases differ only in the value of the total density fluctuation, defined as the volume average $\sigma_{n}=<\tilde{n}^{2} / n^{2}>1 / 2$, which is equal to $2.5 \times 10^{-3}, 5.0 \times 10^{-3}$ and $1.0 \times 10^{-2}$, respectively. These results show that a rise in the level of plasma fluctuations causes a spectral broadening of the reflected waves and a decrease in the amplitude $\left(A_{0}\right)$ of the wave propagating along the direction of specular reflection.

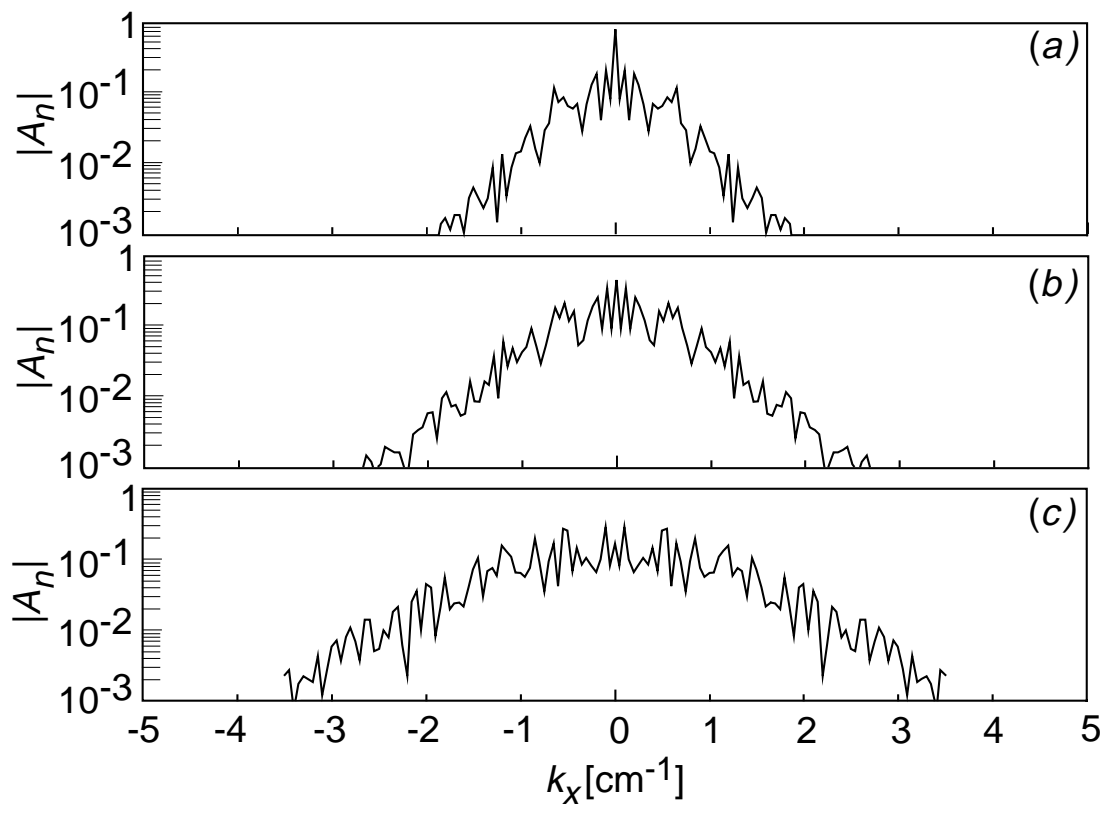

FIG. 2. Spectra of backward waves as a function of $k_{x}$ for fluctuations with $\Delta k_{x}=0.5 \mathrm{~cm}^{-1}, \Delta k_{r}=1 \mathrm{~cm}^{-1}$, and $\sigma_{n}=2.5 \times 10^{-3}$ (a), $\sigma_{n}=5.0 \times 10^{-3}$ (b), $\sigma_{n}=1.0 \times 10^{-2}$ (c). Calculations were done with $N=80$. 
For the same three cases of Fig. 2, Fig. 3 shows the modulus ( $\rho=\left|E_{b}\right|$ ) and the phase deviation $(\tilde{\phi})$ from the mean phase of the backward field at the plasma boundary $\left(r=r_{b}\right)$. These results show large fluctuations in $\rho$ and therefore that the backward field is far from being approximated by a plane wave. Shown in Fig. 3 is also the phase obtained from Eq. (1) neglecting the bending of rays, which in the following we will indicate with $\phi_{G O}$ and refer to as the phase of 1D geometric optic. These results show that $\tilde{\phi}$ is significantly different from $\phi_{G O}$, and that the discrepancy grows with the level of fluctuations.
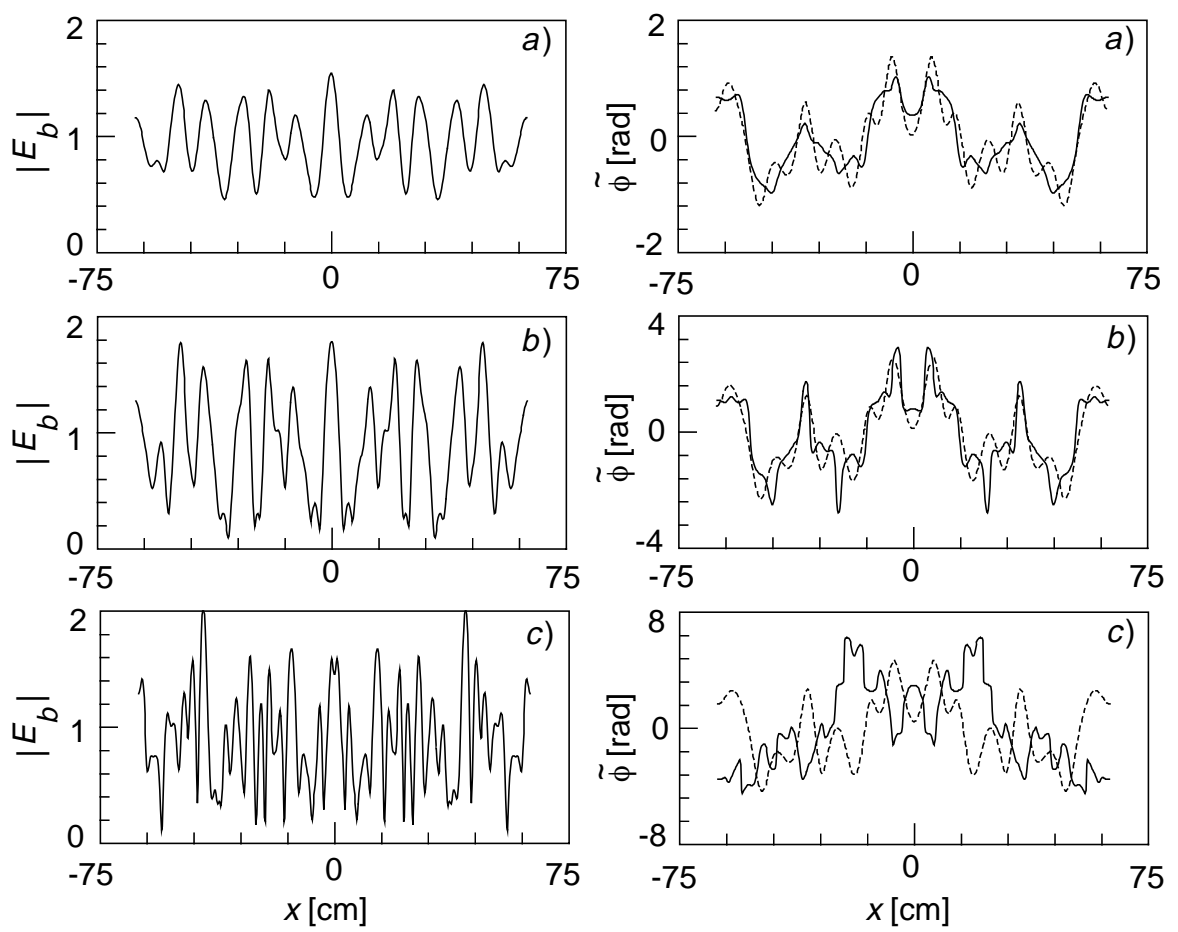

FIG. 3. Modulus $\rho$ of the backward field (left), and fluctuating phase $\tilde{\phi}$ (right) at plasma boundary $\left(r=r_{b}\right)$ for the three cases of Fig. 2. Dashed line is the phase of 1D geometric optics $\left(\phi_{G O}\right)$.

The large fluctuations in the amplitude of the backward field shown in Fig. 3 are caused by the interference of reflected waves. The question, then, is whether the fluctuations in $\rho$ are smaller at other locations. In 
the random phase screen model of reflectometry [6], where the primary effect of plasma fluctuations is a phase modulation of the probing wave near $r \approx r_{c}$, the fluctuations in $\rho$ must be very small near the cutoff.

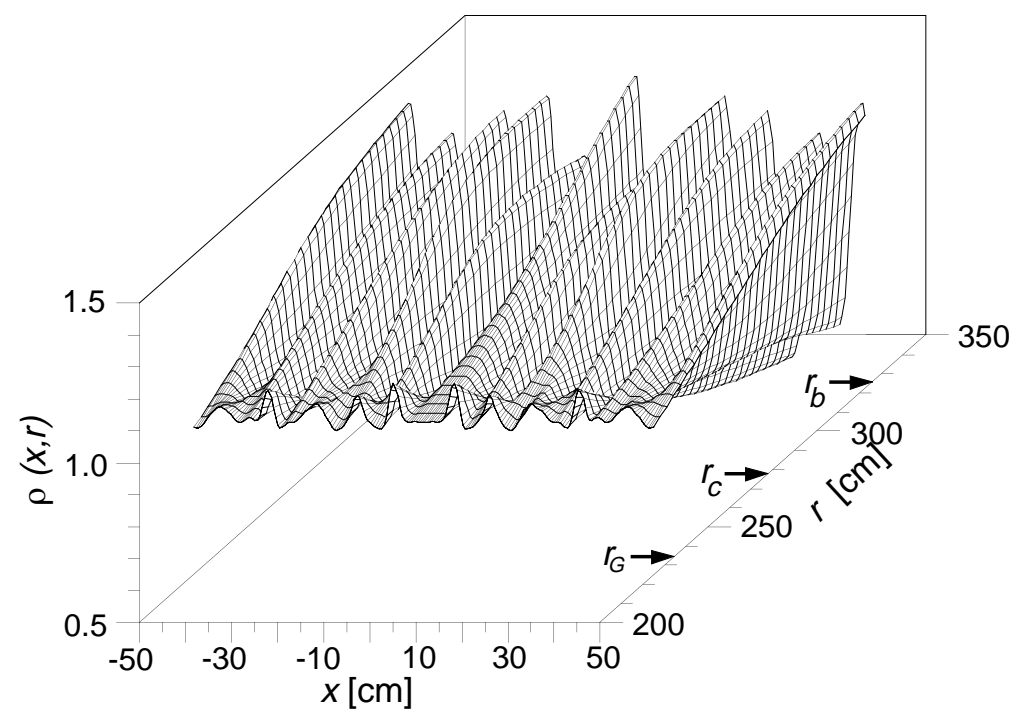

FIG. 4. Isometric 3D plot of the field amplitude $\rho(x, r)$ for the case (a) of Fig. 2; the probing wave is launched from the free-space region $r>r_{b}$.

The isometric 3D plot of $\rho(x, r)$, shown in Fig. 4 for one of the cases of Fig. 2, proves that indeed a region with very small fluctuations exists, but it is located at a considerable distance from the cutoff.

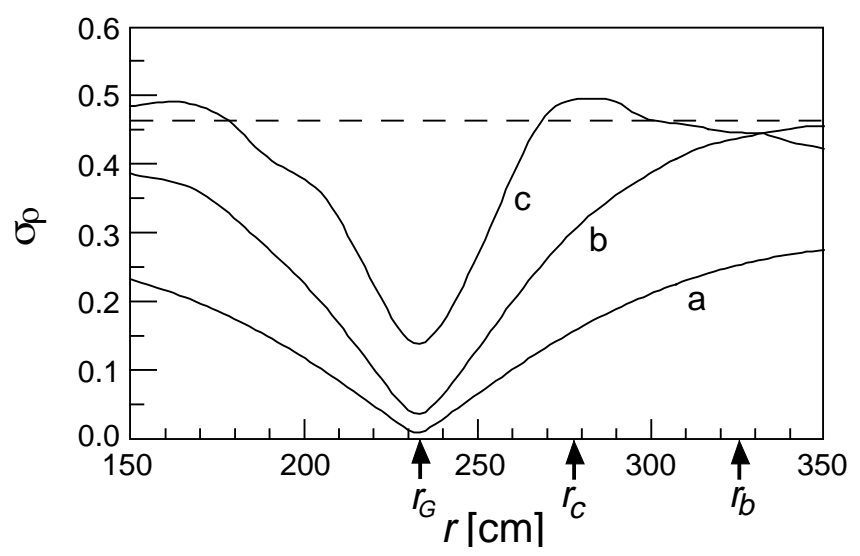

FIG. 5. Value of $\sigma_{\rho}$ as a function of $r$ for the three cases of Fig. 2. The dash line represents the value of $\sigma_{\rho}$ from the Raileigh distribution. 
As a measure of the fluctuation of $\rho(x, r)$, we define the variance $\sigma_{\rho}^{2}(r)=<\left(\rho-<\rho>_{r}\right)^{2}>_{r}$, where $<\cdots>_{r}$ indicates the average at constant value of $r$. Figure 5 shows that the radius $r_{G}$ of the absolute minimum of $\sigma_{\rho}$ is the same for all the cases of Fig. 2. It is also interesting to note that for $r>r_{G}$, Fig. 5 shows that $\sigma_{\rho}$ approaches the variance given by the Rayleigh distribution of a Gaussian noise, indicating that as in Fig. 1 the real and the imaginary component of the backward field become two independent normal random variables with zero mean.

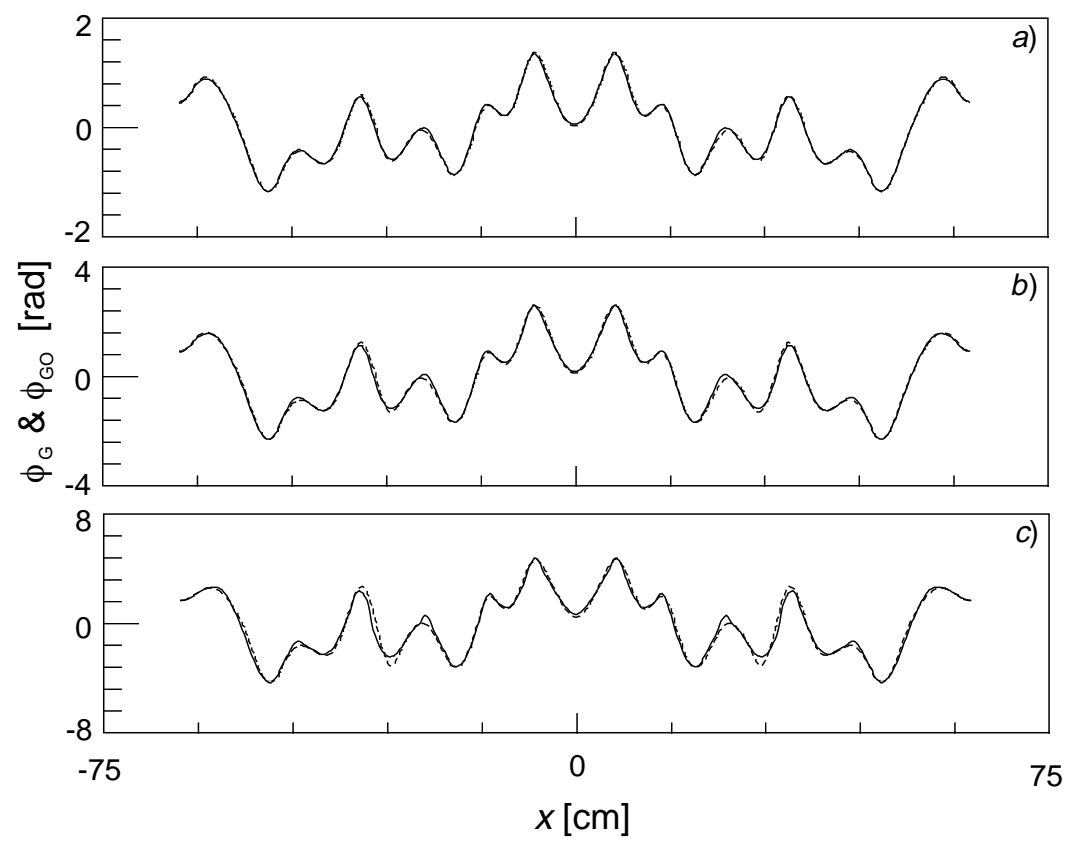

FIG. 6. Fluctuating phases $\phi_{G}$ (solid line) and $\phi_{G O}$ (dashed line) for the three cases of Fig. 2.

Shown in Fig. 6 is the value of $\phi_{G O}$ and the phase deviation from the mean for the backward field at $r=r_{G}$ (in the following referred to as the phase $\left.\phi_{G}\right)$. The excellent agreement between $\phi_{G O}$ and $\phi_{G}$ is tantamount to a reduction of the problem of $2 \mathrm{D}$ fluctuations to that of 1D fluctuations. In both cases, Eq. (2) provides a link between plasma fluctuations and a measurable phase - the signal phase for 1D 
fluctuations and the phase $\phi_{G}$ of the backward field for 2D fluctuations.

The two properties of the plane $r=r_{G}$, i.e., where $\sigma_{\rho}$ has its absolute minimum and $\phi_{G} \approx \phi_{G O}$, would make the backward field appear to an observer in free-space as coming from a virtual location behind the cutoff. This is the result of the spatial variation of $\varepsilon_{0}$ that causes a bending towards the $r$-axis in the trajectory of waves with $k_{x} \neq 0$. To quantify this phenomenon, let us consider the geometry of Fig. 7 where the plasma is divided into $N$ plane slabs with thickness $\delta$ and uniform permittivity $\varepsilon_{i}$. For a ray with wave number $k_{x}$, we can easily derive the difference equation

$$
l_{i+1}-l_{i}=l_{i} \frac{\tan \left(\theta_{i}\right)}{\tan \left(\theta_{i+1}\right)}-l_{i}+\delta,
$$

where $R_{i}$ and $\theta_{i}$ are the radius and the angle of intersection with the $r$ axis of the ray tangent, and $l_{i}=r_{i}-R_{i}$.

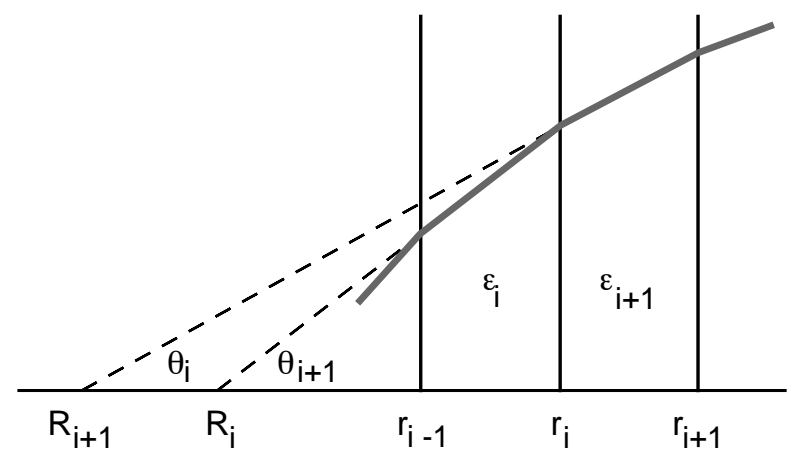

FIG. 7. Geometry used for deriving Eq. (9).

From this and the fact that $k_{x}$ is constant, we get

$$
\frac{l_{i+1}-l_{i}}{\delta} \approx \frac{l_{i}}{\sqrt{\varepsilon_{i}-\alpha}} \frac{\sqrt{\varepsilon_{i+1}-\alpha}-\sqrt{\varepsilon_{i}-\alpha}}{\delta}+1,
$$

where $\alpha=\left(k_{x} / k_{0}\right)^{2}$. The limit $N \rightarrow \infty$ turns Eq. (10) into the differential equation

$$
\frac{d}{d r} \frac{l(r)}{\sqrt{\varepsilon_{0}(r)-\alpha}}=\frac{1}{\sqrt{\varepsilon_{0}(r)-\alpha}},
$$


which, apart from terms of order $\alpha L_{\varepsilon}$, gives

$$
l\left(r=r_{b}\right) \approx \int_{r_{c}}^{r_{b}} \frac{1}{\sqrt{\varepsilon_{0}(r)}} d r .
$$

Since this expression does not depend on $k_{x}$, all rays starting from the same point will appear as coming from the radial position

$$
r=r_{G} \approx r_{b}-\int_{r_{c}}^{r_{b}} \frac{1}{\sqrt{\varepsilon_{0}(r)}} d r .
$$

It is interesting to note that the integral in this equation is the group delay multiplied by the speed of light.

Figure 8, where $\sigma_{\rho}(r)$ is displayed for three density profiles having the same cutoff radius, shows that Eq. (13) agrees with the results of the numerical simulations.
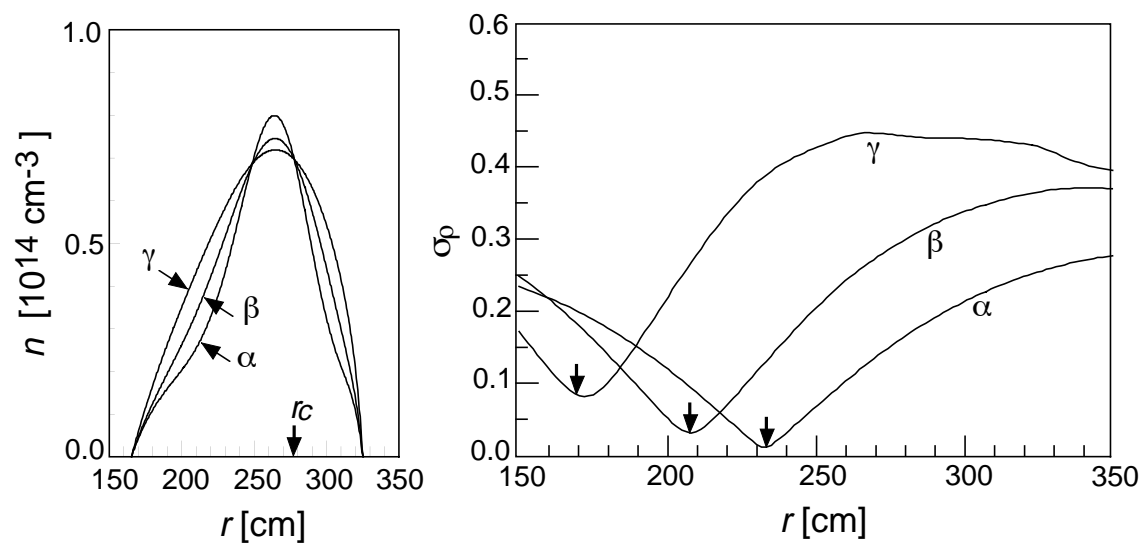

FIG. 8. Value of $\sigma_{\rho}$ (right) for three density profiles (left) having identical cutoffs ( $\alpha$ is the profile used throughout the paper). Fluctuations are as in case $(a)$ of Fig. 2. Arrows on the right graph indicate the value of $r_{G}$ from Eq. (13).

In deriving Eq. (12) we have neglected terms of order $L_{\varepsilon}\left(k_{x} / k_{0}\right)^{2}$, which is also the magnitude of the displacement of the wave turning point from the cutoff radius. Since these terms depend on the value of $k_{x}$, their inclusion would make $r_{G}$ depend on $k_{x}$ as well. This explains why the minimum value of $\sigma_{\rho}$ increases with $\sigma_{n}$ (i.e., with $\Delta k_{x}$ ) as in Fig. 5, or with $L_{\varepsilon}$ as in Fig. 8. As we shall soon see in Sec 4, this sets 
the conditions for the applicability of the method proposed in the paper.

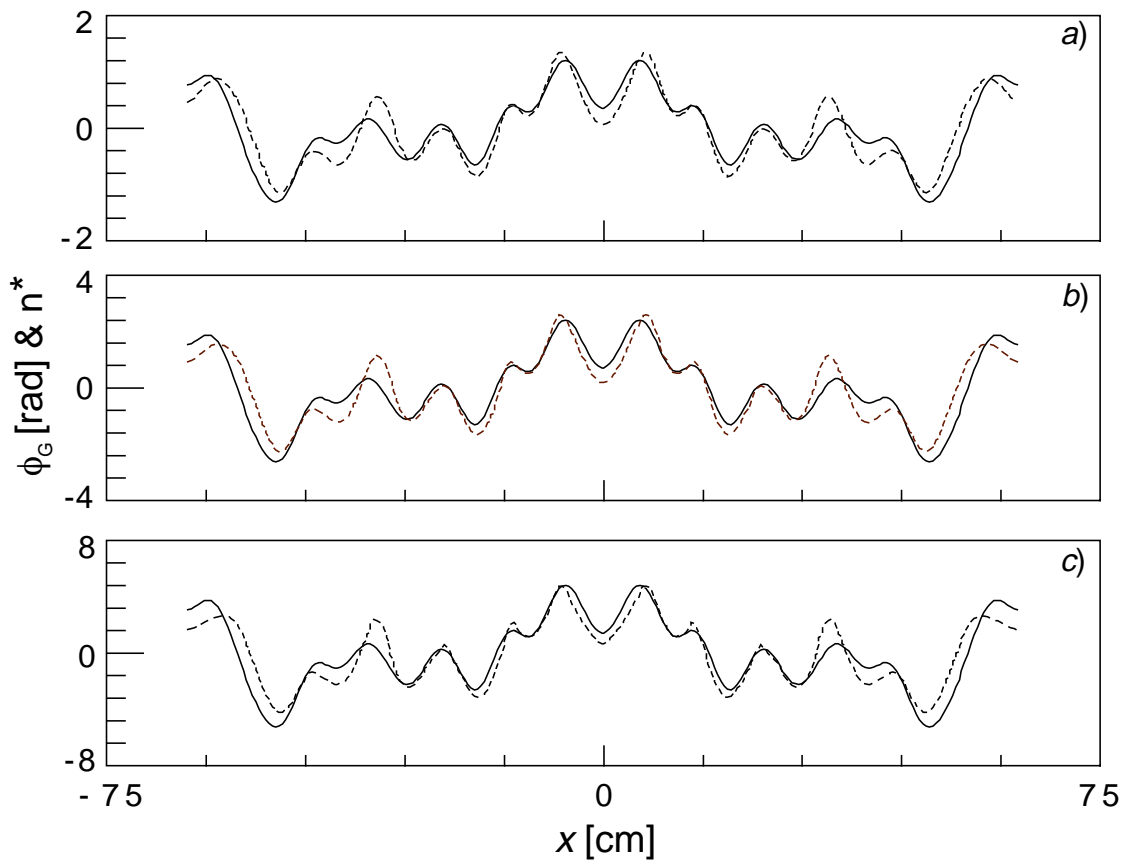

FIG. 9. Phase $\phi_{G}$ (dash line) and normalized density fluctuation $n *$ at $r=r_{c}+0.8 \mathrm{~cm}$ (solid line) for the three cases of Fig. 2.

The results described so far represent an enormous reduction in the difficulty of obtaining the spectrum of turbulent fluctuations from reflectometry data. However, as explained in the introduction, we are looking for an experimental technique capable of providing a direct visualization of turbulent fluctuations. Thus the crucial issue is the similarity of the phase $\phi_{G}$ with the local value of density fluctuations near the cutoff. This is illustrated in Fig. 9, again for the three cases of Fig. 2, where $\phi_{G}$ is shown together with the normalized fluctuation $n^{*} \equiv\left(\sigma_{\phi} / \sigma_{n}\right)(\tilde{n} / n)$, with the factor $\sigma_{\phi} / \sigma_{n}=\left(\pi^{3 / 2} k_{0}^{2} L_{n} / \Delta k_{r}\right)^{1 / 2}$ given by Eqs. (2)-(4). In Fig. 9, we use the radius $r=r_{c}+0.8 \mathrm{~cm}$ that gives the best agreement between $n^{*}$ and $\phi_{G}$. This radial position is shifted from $r_{c}$ by an amount of order $\left(k_{0} L_{\varepsilon}\right)^{1 / 3} / k_{0}$, i.e., it is located where waves with $\left|k_{x}\right|<<k_{0}$ have the largest amplitude. The small 
discrepancy between $\phi_{G}$ and $n *$ is due to the different structure of these quantities, the former being an integral function of the second. This is also what produces the different dependence on $k_{r}$ of their power spectra (Eq. (2)).

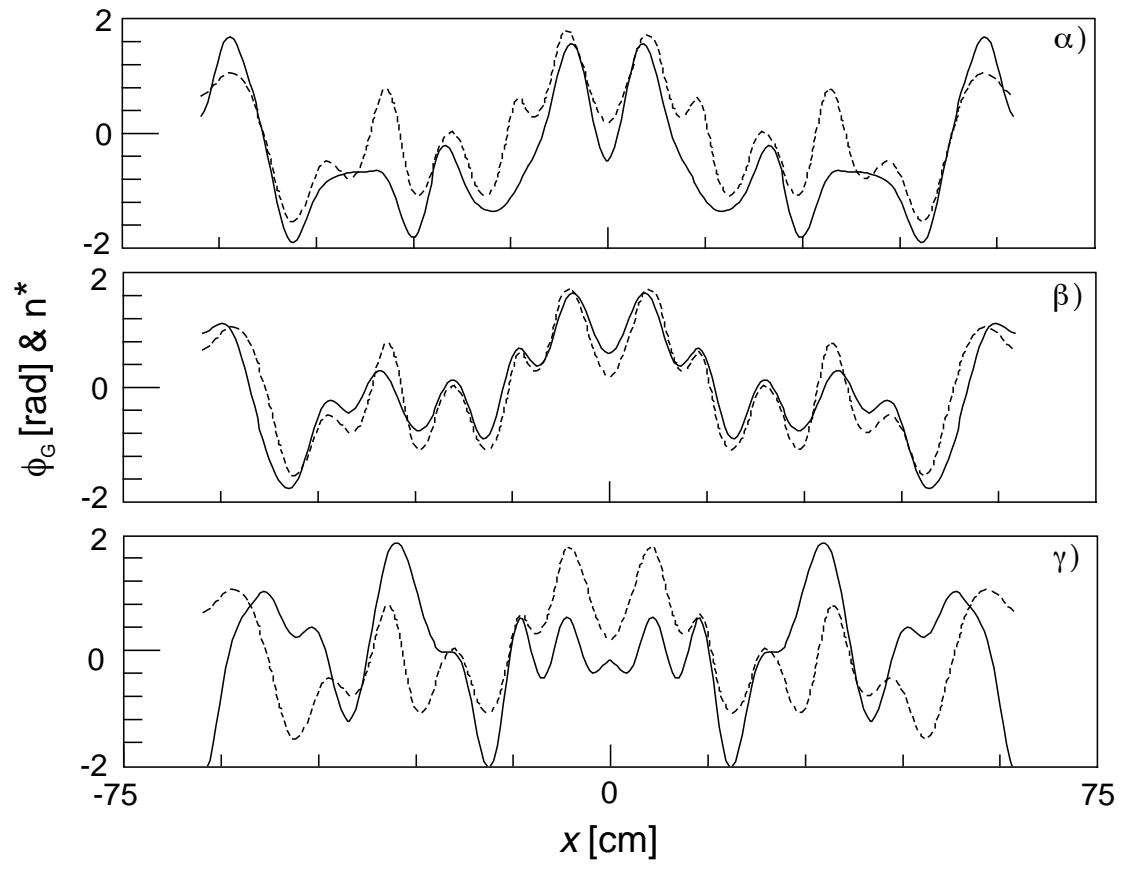

FIG. 10. Phase $\phi_{G}$ (dashed line) and normalized density fluctuations $n^{*}$ (solid line) for $r=r_{c}(\alpha), \quad r=r_{c}+0.8(\beta)$ and $r=r_{c}+1.8(\gamma)$. Fluctuations are as in case $(b)$ of Fig. 2 but with $\Delta k_{r}=2 \mathrm{~cm}^{-1}$.

The similarity between $n *$ and $\phi_{G}$ degrades very quickly away from the optimal position. This is illustrated in Fig. 10, which shows the phase $\phi_{G}$ and the normalized density $n^{*}$ at three radial locations for fluctuations similar to those in case $(b)$ of Fig. 2 but with the larger $\Delta k_{r}=2.0 \mathrm{~cm}^{-1}$, corresponding to a radial correlation length of approximately $1 \mathrm{~cm}$. Note that the optimum radius is the same as in Fig. 9, with deviations that are similar in spite of the shorter radial scale of density fluctuations. Thus the conclusion that the measurement of the phase $\phi_{G}$ can provide the local value of plasma density fluctuations near the cutoff. 


\section{Discussion}

The results in the previous section could be summarized by saying that the field of reflected waves arises near the cutoff from the phase modulation of the probing wave, with a magnitude given by $1 \mathrm{D}$ geometric optics, i.e., neglecting the effects of fluctuations on ray trajectories. Because of the distorting effect of the non-uniformity of the average plasma permittivity $\left(\varepsilon_{0}\right)$, the backward field appears to arrive from a distant point $\left(r_{G}\right)$ behind the cutoff, where it can be approximated by $E=\exp [i \tilde{\phi}(x)]$. Assuming that the phase $\tilde{\phi}$ is a normal random variable with mean $<\tilde{\phi}>=0$, variance $\sigma_{\phi}^{2} \equiv<\tilde{\phi}^{2}>$ and autocorrelation $\gamma_{\phi}(\xi) \equiv<\tilde{\phi}_{1}(x) \tilde{\phi}_{2}(x+\xi)>/ \sigma_{\phi}^{2}$, for the first two moments of the backward field we obtain $<E>=\exp \left(-\sigma_{\phi}^{2} / 2\right)$ and $<E_{1} E_{2}^{*}>=\exp \left[-\sigma_{\phi}^{2}\left(1-\gamma_{\phi}\right)\right]$, which are both decreasing functions of $\sigma_{\phi}$. Consequently, in agreement with results in Fig. 2, as the level of fluctuations increases, the amplitude of the wave propagating along the direction of specular reflection (i.e., $<E>$ ) decreases, and the spectrum of reflected waves (i.e., the Fourier transform of $\left\langle E_{1} E_{2}^{*}>\right.$ ) broadens. In particular, for $\sigma_{\phi}>>1$, taking $\gamma_{\phi}(\xi)=\exp \left[-(\xi / \delta)^{2}\right]$ and expanding to the second order in $\xi$, we obtain $<E_{1} E_{2}^{*}>\approx \exp \left[-\left(\sigma_{\phi} \xi / \delta\right)^{2}\right]$. Thus, away from the cutoff, if $\Delta k_{x}$ is the spectral width of fluctuations and $\sigma_{\phi} \Delta k_{x}<<k_{0}$, the reflected waves are distributed over a range of radial wave numbers $\delta k_{r} \approx \sigma_{\phi}^{2} \Delta k_{x}^{2} / 2 k_{0}$. Consequently, at a distance from $r=r_{G}$ that is larger than the diffraction distance $D=1 / \delta k_{r}$, the interference of waves will produce (as in Figs. 1 and 3) a complicated field pattern with large amplitude variations and random phases. This suggests that the amplitude $\rho$ of the measured signal must follow the distribution derived by Rice [7] for a signal containing a sinusoidal coherent component and a Gaussian noise, which is given by 


$$
P(\rho)=\frac{\rho}{\sigma^{2}} \mathrm{e}^{-\left(\rho^{2}+\rho_{0}^{2}\right) / 2 \sigma^{2}} I_{0}\left(\frac{\rho \rho_{0}}{\sigma^{2}}\right)
$$

where $I_{0}$ is the modified Bessel function of order zero, $\sigma^{2}$ is the variance of both the real and imaginary parts of the Gaussian noise, and $\rho_{0}$ is the amplitude of the sinusoidal signal. In the case of our numerical simulation, since we use a probing wave with unit amplitude, $\sigma^{2}=\left(1-\rho_{0}^{2}\right) / 2$ and $\rho_{0}^{2} \approx \exp \left(-\sigma_{\phi}^{2}\right)$. The Rice distribution becomes a Rayleigh distribution for $\sigma_{\phi}>>1$, in agreement with the experimental results of Fig. 1. A statistical analysis of several numerical realizations of $E_{b}$ indicates that Eq. (14) is in good agreement with the results of the numerical simulations [5].

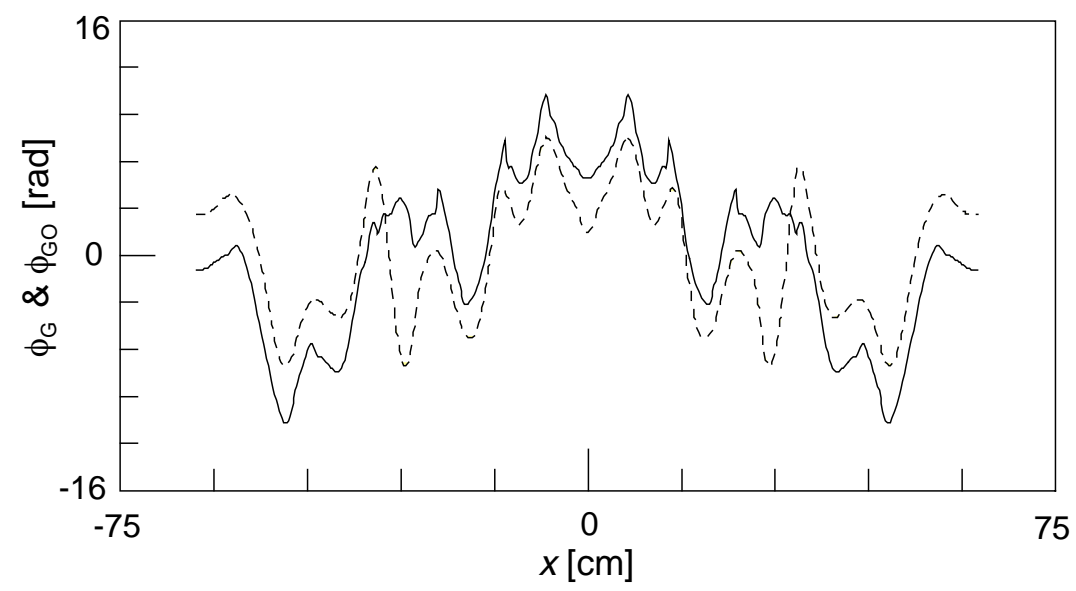

FIG. 11. Fluctuating phases $\phi_{G}$ (solid line) and $\phi_{G O}$ (dashed line) for $\sigma_{n}=2.0 \times 10^{-2}$ and other parameters in Fig. (2).

The model of reflectometry suggested by the numerical results of Sec. 3 will obviously fail for large amplitude fluctuations. In fact, since each spectral component of the backward field originates near the corresponding turning point, this must occur when the turning points are distributed over a distance $\Delta r_{c}$ that is comparable to the radial scale length of fluctuations $1 / \Delta k_{r}$, i.e., when $\Delta k_{r} \Delta r_{c}>1$. Since $\Delta r_{c} / L_{\varepsilon} \approx \sigma_{\phi}^{2} \Delta k_{x}^{2} / k_{0}^{2}$, we conclude that a necessary condition for the validity of the above model of reflectometry is $\sigma_{\phi}^{2}<k_{0}^{2} / L_{\varepsilon} \Delta k_{r} \Delta k_{x}^{2}$. By 
expressing $\sigma_{\phi}^{2}$ in terms of $\sigma_{n}^{2}$ (Eqs. (2)-(4)), this condition can be cast in the form

$$
\sigma_{n}^{2}<\frac{1}{\pi^{3 / 2} L_{n}^{2} \Delta k_{x}^{2}} .
$$

For the plasma parameters in Fig. 2 (where $L_{n}=50 \mathrm{~cm}$ ), Eq. (15) gives $\sigma_{n}<1.7 \times 10^{-2}$, which explains why in all three cases of Fig. 6 we obtained $\phi_{G} \approx \phi_{G O}$.

Figure 11 shows the effect of doubling the value of $\sigma_{n}\left(2.0 \times 10^{-2}\right)$ for case (c) of Fig. 6, making the amplitude of fluctuations larger than the limit imposed by Eq. (15). As expected, this results in a large discrepancy between $\phi_{G}$ and the phase of geometric optics $\phi_{G O}$.

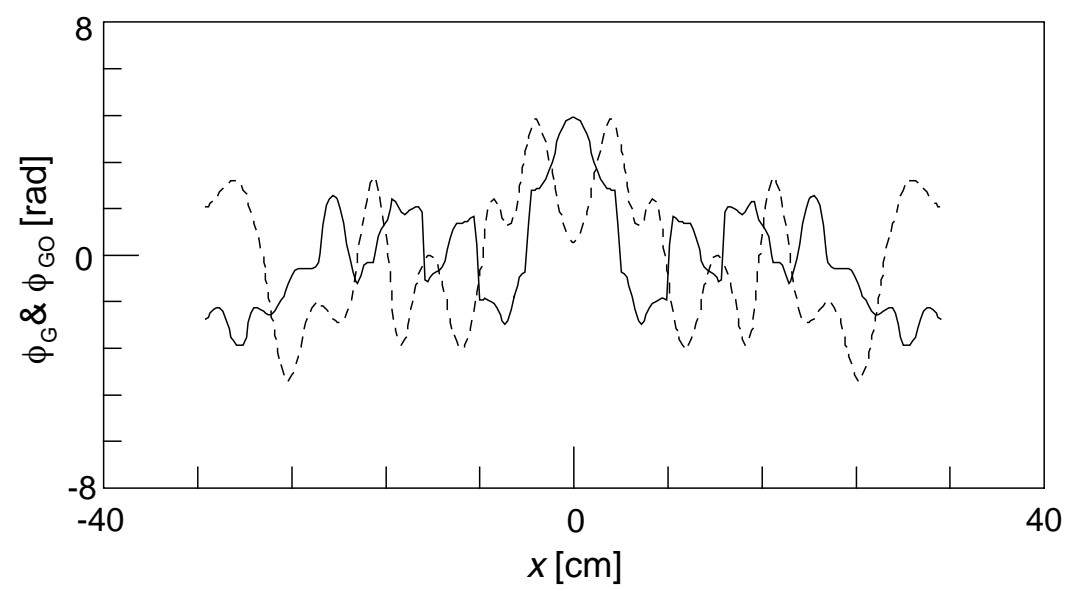

FIG. 12. Fluctuating phases $\phi_{G}$ (solid line) and $\phi_{G O}$ (dashed line) for $\Delta k_{x}=1 \mathrm{~cm}^{-1}$ and other parameters of case (c) in Fig. (6).

Another way of violating Eq. (15) is by increasing the spectral width of the fluctuations. This is illustrated in Fig. 12 showing the result of doubling the value of $\Delta k_{x}$ for case (c) of Fig. 8. Since this pushes the limit imposed by Eq. (15) $\left(\sigma_{n}<0.8 \times 10^{-2}\right)$ below the level of plasma fluctuations, the result is again a large discrepancy between $\phi_{G}$ and $\phi_{G O}$.

These results show how quickly $\phi_{G}$ departs from $\phi_{G O}$ when the condition of Eq. (15) is violated. In tokamaks, both theory and 
experiments indicate that the amplitude of short-scale density fluctuations obeys the mixing length criterion $\sigma_{n}<1 / k_{r} L_{n}$ [1]. From this and Eq. (15), we conclude that a condition for the applicability of geometric optics to reflectometry measurements of 2D plasma fluctuations in tokamaks is $\Delta k_{r}>\pi^{3 / 4} \Delta k_{x}$ (besides the condition $\Delta k_{r}<k_{0} /\left(k_{0} L_{\varepsilon}\right)^{1 / 3}$ that is necessary for 1D fluctuations as well).

\section{Imaging Reflectometry}

The numerical results of the previous sections emphasize the importance of measuring $\tilde{\phi}_{G}$ for obtaining the value of density fluctuations near the cutoff. In principle, this could be done by collecting the reflected waves with a wide aperture antenna, and by imaging the cutoff onto the detector plane (taking the average plasma permittivity into account). This is the first novelty of the scheme proposed in this paper. A second novelty is the simultaneous sampling of a large portion of a magnetic surface, which requires the use of large microwave beams and 2D arrays of detectors. The latter are technically feasible, as shown in Ref. [8] which describes a microwave camera with a focal plane array (4 rows of 64 elements) for the detection of the human body emission at $94 \mathrm{GHz}$ with a resolution of $1 \mathrm{~K}$ and a frame rate of $30 \mathrm{~Hz}$. Another example can be found in Ref. [9] which describes the measurement of the electron cyclotron emission in the TEXT tokamak using a wide band 20 channel array in the frequency range 90-110 GHz. More recently, similar measurements have been repeated in the RTP tokamak [10] using a 16 channel array in the range $100-140 \mathrm{GHz}$.

Figure 13 illustrates the conceptual design of a microwave imaging reflectometer for the visualization of turbulence in a tokamak plasma. In this scheme, as in the numerical simulations, the rays of the probing wave impinge perpendicularly upon the cutoff surface. This is obtained by using two cylindrical lenses ( $L_{1}$ and $L_{2}$ ) with different focal points. For the case considered in Fig. 13, where the probing wave has a 
frequency of $120 \mathrm{GHz}$ and the $\mathrm{X}$-mode of propagation, the focal point of $L_{2}$ is at $R=2.3$, while that of $L_{1}$ is obviously at $R=0$. Since the former depends on the wave frequency, the position of $L_{2}$ must be adjustable. Outside of the plasma, then, the backward wave is reflected by the semitransparent reflector $M$ and an image of the cutoff is formed by the spherical lens $L_{3}$ onto the plane $P$, where the field is measured with a $2 \mathrm{D}$ array of microwave receivers.

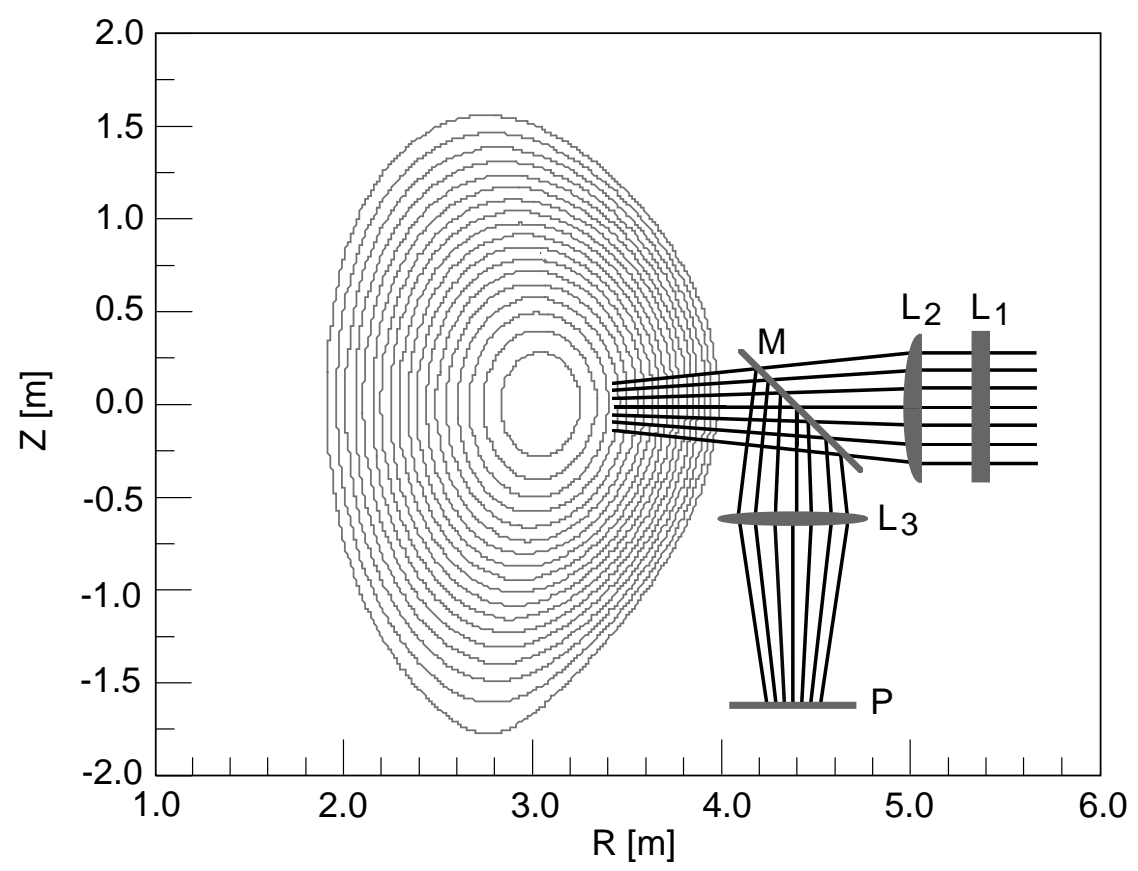

FIG. 13. Conceptual design of an imaging reflectometer for a JET-like tokamak; $L_{1}$ and $L_{2}$ are cylindrical lenses, $L_{3}$ is a spherical lens, $M$ is a semitransparent mirror, and $P$ is the detector plane.

In Fig. 13, the function of the cylindrical lenses $L_{1}$ and $L_{2}$ is to tailor the wave front of the probing wave to the shape of the cutoff surface, which allows the mapping of the cutoff onto the detector plane by the spherical lens $L_{3}$. Figures 14 and 15 show the result of replacing $L_{1}$ and $L_{2}$ with a single spherical lens and optimizing the ray trajectories on either the poloidal (Fig. 14) or the toroidal (Fig. 15) 
plane. In both cases, $L_{3}$ would not be able to create an image of the reflecting cutoff onto the detector plane. Thus, the use of the cylindrical lenses $L_{1}$ and $L_{2}$ is to combine the poloidal ray trajectories in Fig. 14 with the equatorial trajectories in Fig. 15.
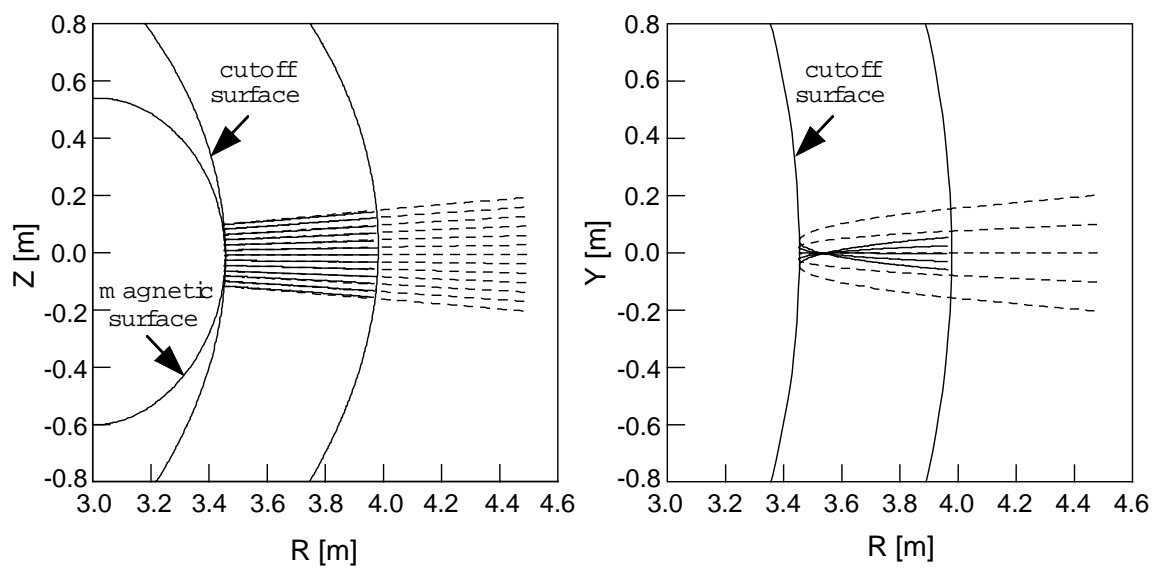

FIG. 14. Ray trajectories (from a ray tracing code) of the probing wave in the absence of fluctuations when the focal length of $L_{1}$ in Fig, 13 is equal to that of $L_{2}$ : dash lines are forward rays and solid lines are reflected rays on the poloidal (left) and equatorial (right) planes.

By probing the plasma using simultaneously multiple waves with closely spaced cutoffs, the proposed method could provide the full 3D structure of fluctuations.
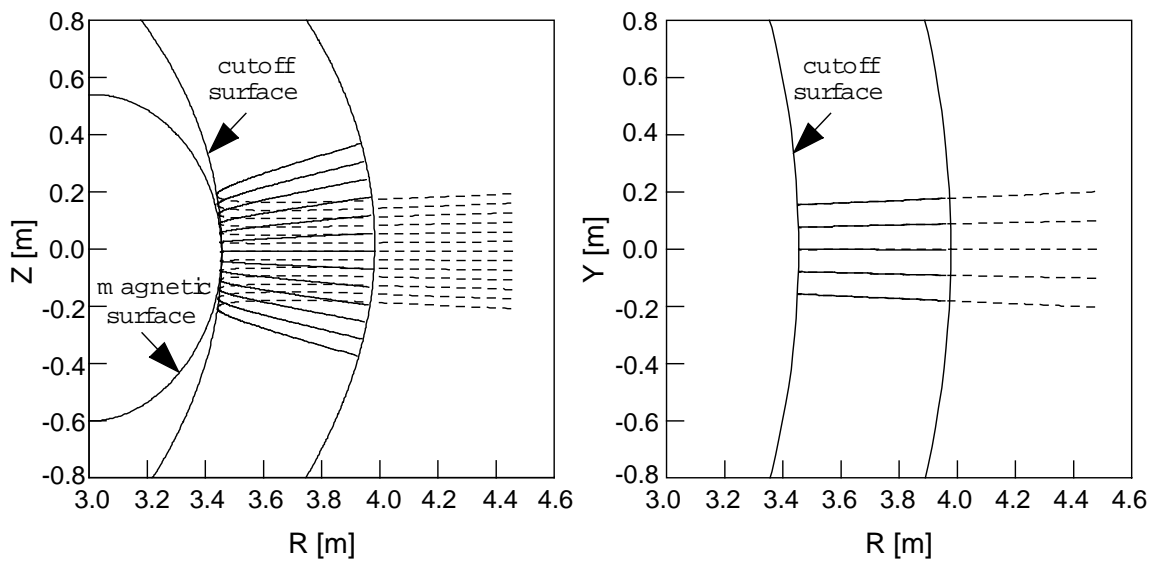

Fig. 15. Same as in Fig. 13 when the focal length of $L_{2}$ in Fig. 13 is equal to that of $L_{1}$. 
Finally, the proposed method can use either the ordinary or the extraordinary mode of wave propagation, but the latter must be preferred because of the better spatial resolution that derives from the larger probing frequency of the extraordinary mode. The spatial resolution is given by $\delta \approx 2\left(\lambda_{0} / D\right)\left(r_{b}-r_{G}\right)$, where $\lambda_{0}=2 \pi / k_{0}$ and $D$ is the diameter of the probing beam. For the case of Fig. 13, we get $\delta \approx 1 \mathrm{~cm}$ with $D=50 \mathrm{~cm}$.

\section{Conclusion}

In conclusion, the method described in this paper is a first attempt at developing techniques for the global visualization of turbulent and coherent structures in tokamak plasmas. Undoubtedly, its practical implementation presents serious difficulties, such as the need for large ports and 2D arrays of microwave detectors. Nevertheless, the proposed technique has the potential for providing new information on the spatial structure of turbulent fluctuations in tokamaks, that could be useful for advancing the theory of plasma turbulence or for checking the results of numerical simulations.

\section{Acknowledgements}

This work was supported by U.S. DOE Contract No. DE-AC02-76CHO-3073

\section{References}

[1] W. Horton, Rev. Mod. Phys. 71, 735 (1999).

[2] P. J. Holmes, et al., Physics Reports 287, 337 (1997).

[3] E. Mazzucato, Rev. Sci. Instrum. 69, 2201 (1998).

[4] E. Mazzucato, et al., Phys. Rev. Lett. 77, 3145 (1996).

[ 5] E. Mazzucato, Rev. Sci. Instrum. 69, 1691 (1998).

[ 6] E. Mazzucato and R. Nazikian, Phys. Rev. Lett. 71, 1840 (1993). 
[ 7] S. O. Rice, Bell System Tech. J., 23, 282 (1944); 24, 96 (1945) (reprinted in N. Wax, Selected Papers on Noise and Stochastic Processes (Dover, New York, 1954)).

[ 8] G. C. Huguenin, SPIE 2938, 152 (1996).

[ 9] R. P. Hsia, et al., Rev. Sci. Instrum. 68, 488 (1997).

[10] B. H. Deng, et al., Rev. Sci. Instrum. 70, 998 (1999). 